\title{
Stress intensification - an abnormal phenomenon observed during stress relaxation of dynamic coordination polymer
}

\author{
N. N. Xia ${ }^{1}$, M. Z. Rong ${ }^{1}$, M. Q. Zhang ${ }^{1 *}$, S.-W. Kuo ${ }^{2}$ \\ ${ }^{1}$ Key Laboratory for Polymeric Composite and Functional Materials of Ministry of Education, GD HPPC Lab, School of \\ Chemistry and Chemical Engineering, Sun Yat-Sen University, 510275 Guangzhou, China \\ ${ }^{2}$ Department of Material and Optoelectronic Science, National Sun Yat-Sen University, 804 Kaohsiung, Taiwan
}

Received 14 January; accepted in revised form 28 March 2016

\begin{abstract}
The authors report that during tensile stress relaxation test of a polyurethane crosslinked by catechol-iron coordination bonds, the stress gradually increases with time after the initial drop, which differs from the documented behaviors of other materials. Based on model experiment and structural characterization, water triggered rapid increase of dynamic crosslinkages accompanied by Poisson's contraction is found to be responsible for the stress intensification effect. In addition, the influential factors are carefully discussed. The findings might help to develop novel polymeric materials with improved application performance under loading conditions.
\end{abstract}

Keywords: smart polymers, stress relaxation, polyurethane, dynamic coordination bond, crosslink

\section{Introduction}

Stress relaxation of materials used to refer to stress decrease with time under constant strain. In the case of polymers, macromolecular conformation has to be at a non-equilibrium state upon being stressed. Accordingly, movement of chain segments would take place to reduce or relieve the internal stress with increasing time.

In reality, the mechanisms involved in stress relaxation fall into five groups depending on polymer species and practical application circumstances [1,2]: (i) chain scission (mostly because of hydrolysis or oxidative degradation), (ii) bond interchange, (iii) viscous flow (caused by slippage of linear chains), (iv) Thirion relaxation (reversible relaxation of physical crosslinks or trapped entanglements in elastomers), and (v) molecular relaxation near glass transition temperature.

From the phenomenological point of view, linear polymers can completely relax stress to zero provided time is long enough, while crosslinked polymers can only relax stress to a certain equilibrium value owing to the network confinement. However, if the crosslinked polymers are built up by reversible bonds, as revealed by recent investigation [3-9], rearrangement of the networks would be allowed when the reversible reaction is triggered. The materials also go to a lower state of energy, leading to complete relaxation of the stress.

Unlike these known behaviors, here we report stress intensification behavior appearing during stress relaxation test of a hyperbranched polyurethane (HBPU), which holds catechol and hydrophilic carboxyl end groups and is crosslinked by catechol- $\mathrm{Fe}^{3+}$ complexes (HBPU-DMPA-[Fe(DOPA) $)_{3}$ ], DMPA = dimethylol propionic acid, DOPA = 3.4-dihydroxyphenyl-L-alanine, refer to Figure 1. That is, after the initial reduction in stress as usual, the stress gradually increases rather than decreases or levels off. Since there is no similar observation in literature, a preliminary exploration of the molecular cause for the abnormal effect 


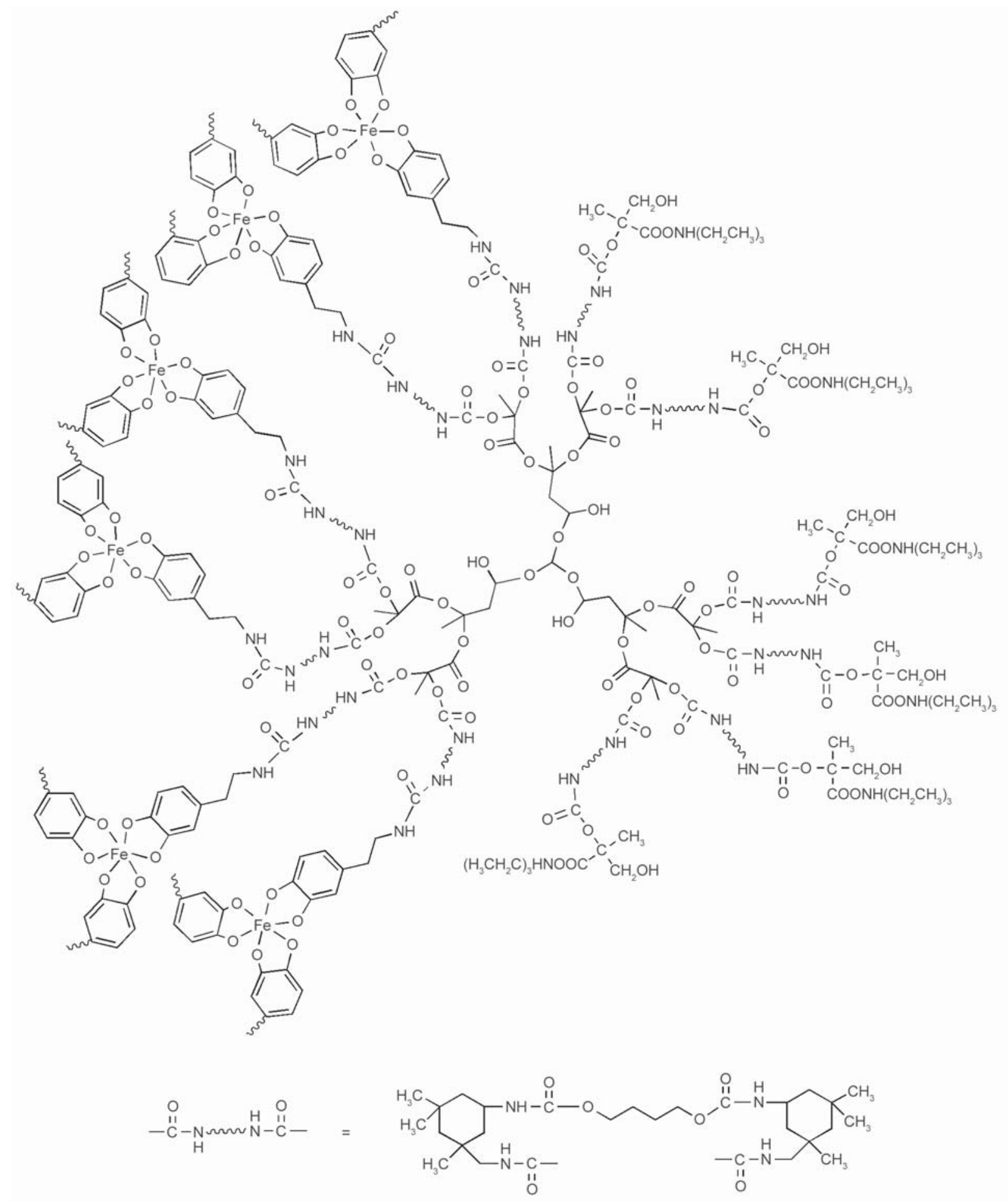

Figure 1. Structure of HBPU-DMPA-[Fe(DOPA $\left.)_{3}\right]$. During the synthesis, hyperbranched polyester is used as the starting material, and then polyurethane prepolymer, DMPA, dopamine hydrochloride, and ferric chloride are introduced, respectively (Figure 2)

is conducted hereinafter to have an in-depth understanding. The dynamic coordination-dissociation behavior of the crosslinkages in the polymer is absent in conventional irreversibly bonded versions. It is hoped that the present work will help to carry forward this interesting property for future design, syn- thesize, manufacturing and application of novel materials.

\section{Experimental}

HBPU-DMPA-[Fe(DOPA $\left.)_{3}\right]$ with $\mathrm{Fe}^{3+} /$ dopamine molar ratio of 1:3 was synthesized following the pro- 
cedures described in ref. [10] (Figure 2). $T_{\mathrm{g}}$ of the soft segments is $-63.3^{\circ} \mathrm{C}$, while that of the hard segments is $-7.5^{\circ} \mathrm{C}$, as determined by dynamic mechanical analysis (DMA) [10].

Stress relaxation was measured at $25^{\circ} \mathrm{C}$ in tensile mode at a constant strain of $10 \%$ with DMA $(01 \mathrm{~dB}$ MetraviB DMA-25N, ACOEM Group, Limonest, France). Prior to the measurement, the specimens were saturated by water with different $\mathrm{pH}$ values. Then, some of the specimens were directly sent for testing (called water saturated specimens hereinafter), while the rest were dried and tested (called dry specimen). During testing the water saturated specimens were wrapped by waterlogged absorbent cotton to maintain water content.

Rheological data were obtained from a strain-controlled ARG2 rheometer (TA Instruments, New Castle, USA) with $25 \mathrm{~mm}$ parallel-plate geometry (disk-<smiles>CC(O)CCCCO</smiles>

PTMEG<smiles>Cc1ccc(O)c(O)c1</smiles>

HBPU-DMPA-DOPA

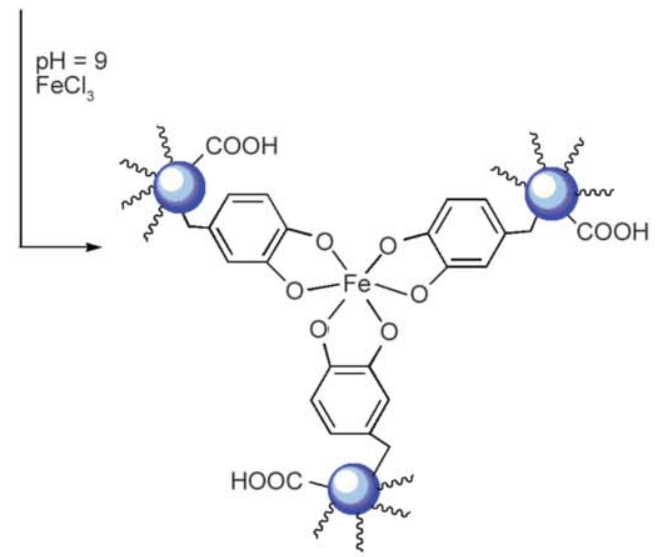<smiles>CC(OCCCCOC(=O)C(C)(C)NC1CC(C)(C)CC(C)(C[N+](=O)[O-])C1)C(=O)NC1CC(C)(C)CC(C)(CN=O)C1</smiles>

PU

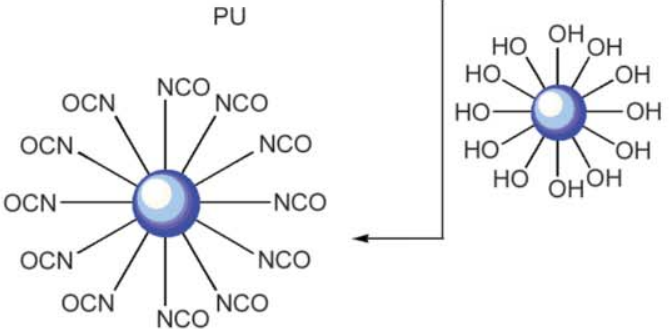

HBPU

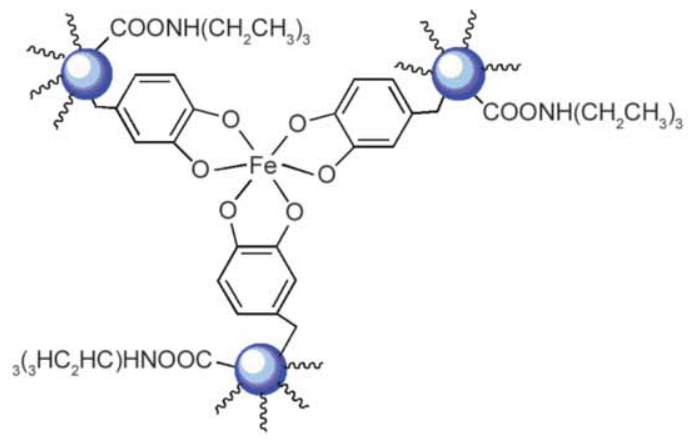

HBPU-DMPA-[Fe(DOPA $\left.)_{3}\right]$

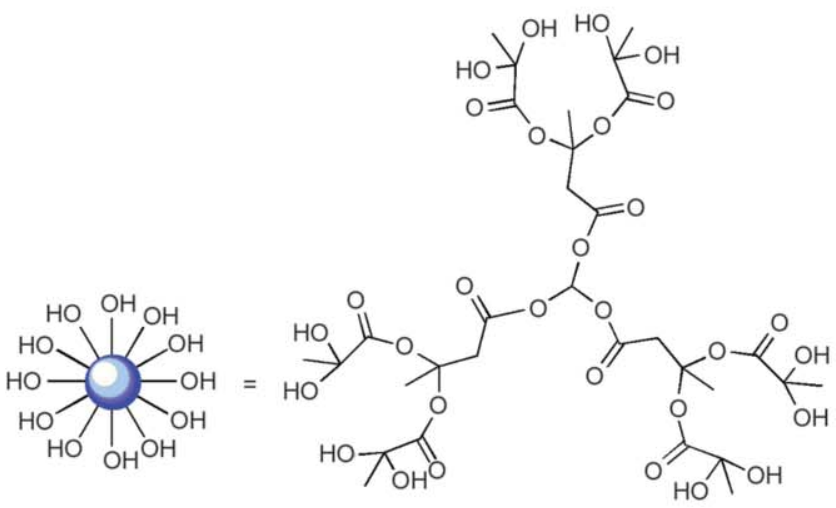

Hyperbranched polyester

Figure 2. Synthesis of HBPU-DMPA-[Fe(DOPA $\left.)_{3}\right]$ 
shaped specimens: $10 \mathrm{~mm}$ in diameter and $2 \mathrm{~mm}$ in thickness). Frequency sweeps at $0.2 \%$ strain were conducted at different temperatures.

To obtain Raman spectra of the materials subjected to volume contraction during tensile stress relaxation, disk-shaped specimens (diameter: $12 \mathrm{~mm}$, thickness: $1.5 \mathrm{~mm}$ ) were pretreated following the above procedures applied for stress relaxation, placed into a mold with round cavity (inner diameter: $12 \mathrm{~mm}$ ) and compressed by a steel cylinder (diameter: $12 \mathrm{~mm}$ ) under $0.6 \mathrm{MPa}$ for preset time. Afterwards, Raman spectra were collected by a confocal Raman microscope (Renishaw inVia, Renishaw, Gloucestershire, UK). The diode-pumped $785 \mathrm{~nm}$ near infrared laser excitation was used in combination with a $20 \times$ microscope objective. The spectra were acquired using an air-cooled CCD behind a grating $\left(300 \mathrm{~g} \cdot \mathrm{mm}^{-1}\right)$ spectrograph with a spectral resolution of $4 \mathrm{~cm}^{-1}$. Because the samples were sensitive to burning by the laser beam, laser power of $10 \mathrm{~mW}$ and exposure time of $20 \mathrm{~s}$ were used for all measurements. Each collected spectrum consisted of 60 accumulations of a $0.2 \mathrm{~s}$ integration time. For each sample, three spectra were collected from different regions and averaged.

\section{Results and discussion}

The polymer HBPU-DMPA-[Fe(DOPA $)_{3}$ ] was originally synthesized for developing lipophilic material capable of self-healing and recycling in seawater $(\mathrm{pH}=8.3)$ [10]. Therefore, characterization of structure and properties is carried out in water as a function of $\mathrm{pH}$ in comparison with that in dry environment.

As shown in Figure 3, the above-mentioned stress intensification can be found under certain circumstances. More exactly, only the specimens of HBPUDMPA- $\left[\mathrm{Fe}(\mathrm{DOPA})_{3}\right]$ saturated by water at $\mathrm{pH}=9$ and 7 exhibit the abnormal manner (Figure 3a). A simple explanation of this habit lies in stress hardening due to improvement of macromolecular chains orientation induced by tension. However, this is an apparent paradox because (i) the small strain applied during stress relaxation is barely enough for chain extension, (ii) the polymers studied here are crosslinked so that the molecular chains are unable to align preferentially along the stretching direction, and (iii) either the same polymer HBPU-DMPA-[Fe(DOPA) 3 , which was firstly saturated by water at $\mathrm{pH}=9$ or 7 and then dried (Fig- ure $3 b$ ), or the control HBPU-DMPA-DOPA (Figure $3 \mathrm{c}$ and Figure 2), behaves like conventional polymers, despite the fact that all the materials shown in Figure 3 were tested on the same conditions.

A careful examination of Figure 3 indicates that (i) water saturation and (ii) higher $\mathrm{pH}$ value of the water are necessary for achieving obvious stress intensification effect of HBPU-DMPA-[Fe(DOPA $\left.)_{3}\right]$. The first issue is precisely the key factor for trigger-
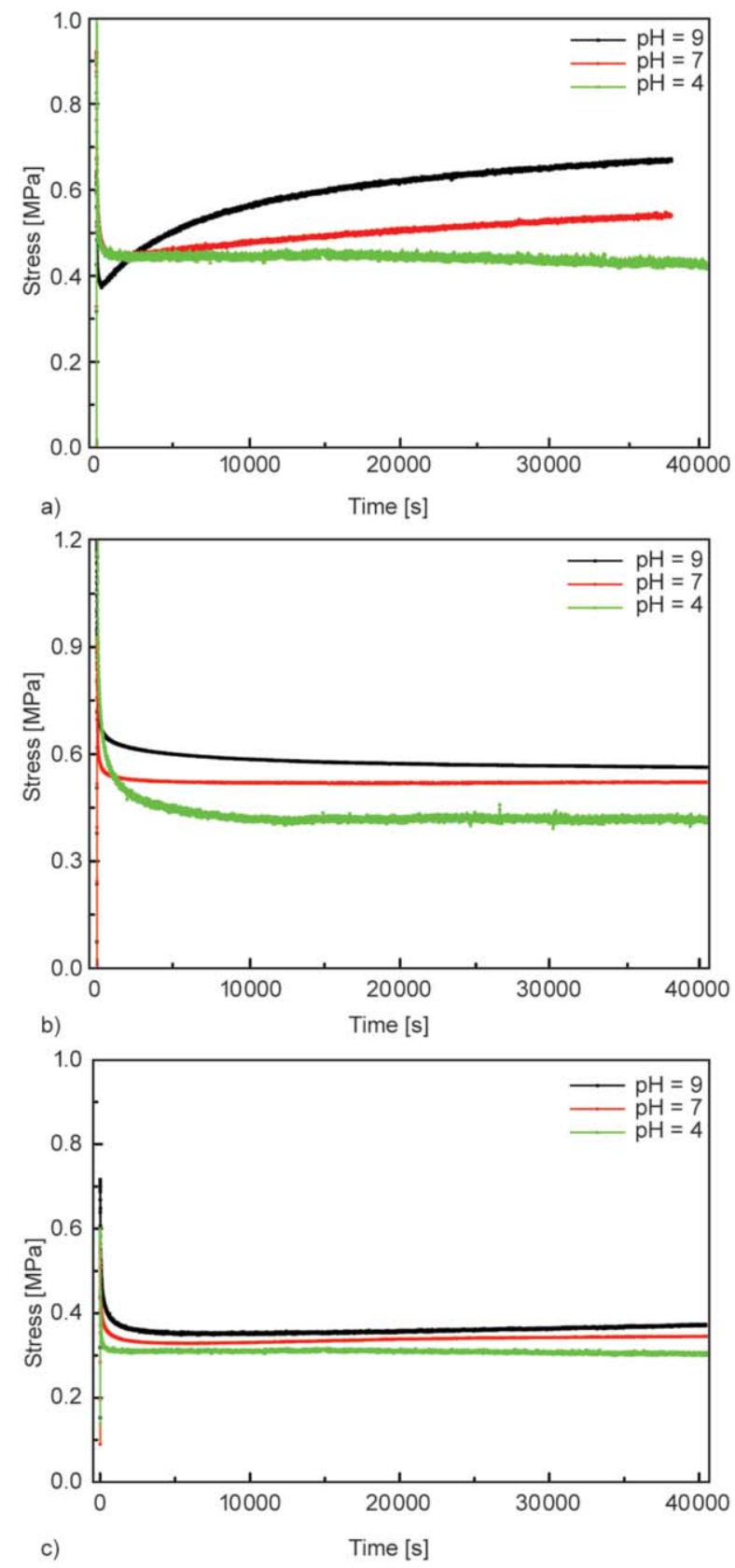

Figure 3. Tensile stress relaxation behaviors of (a) water saturated and (b) dry HBPU-DMPA-[Fe(DOPA $\left.)_{3}\right]$ as a function of $\mathrm{pH}$ in comparison with (c) the water saturated control HBPU-DMPA-DOPA. Temperature: $25^{\circ} \mathrm{C}$. 
ing the dynamic coordinate bonds in HBPU-DMPA$\left[\mathrm{Fe}(\mathrm{DOPA})_{3}\right][10]$. It has been known that when the polymer is immersed in water with different $\mathrm{pH}$ values $\left(9,7\right.$ and 4), tris-, bis- and mono-catechol- $\mathrm{Fe}^{3+}$ coordinations appear in the polymer network successively [11]. Higher coordination number is acquired at higher $\mathrm{pH}$. Moreover, our recent investigation reveals that the DOPA-iron complexation can be triggered to a dynamic state in the presence of water and the dynamic manner is immobilized after removing water. That is, the catechol- $\mathrm{Fe}^{3+}$ crosslinkages of HBPU-DMPA-[Fe(DOPA $\left.)_{3}\right]$ are dynamically connected and disconnected at any time in wet environment. Accordingly, the polymer is in the dynamic equilibrium (in the presence of water) and stable state (in the absence of water), respectively.

By taking advantages of the dynamic reversible crosslinking, the network chains of HBPU-DMPA$\left[\mathrm{Fe}(\mathrm{DOPA})_{3}\right]$ is able to be ceaselessly rearranged. As a result, the frequency dependence of storage shear modulus, $G^{\prime}$, of the water saturated specimen intersects with that of loss shear modulus, $G^{\prime \prime}$, suggesting a transition from elastic-like $\left(G^{\prime}>G^{\prime \prime}\right)$ to viscous-like $\left(G^{\prime}<G^{\prime \prime}\right)$ with decreasing frequency (Figure $4 \mathrm{a}$ ). Since the disconnected networks need time to be reconnected, the system has to perform like viscous fluid at low frequency regime [12-14]. In contrast, the dry HBPU-DMPA-[Fe(DOPA) $)_{3}$ (Figure $4 \mathrm{~b}$ ) and the control without $\mathrm{Fe}^{3+}$ (Figure 4c) do not exhibit the same network reconfiguration due to lack of the dynamic bonds.

Just because of existence of the dynamic reversible bonds, the crosslinked networks should be rearranged to a less stretched state upon being stressed and the water saturated HBPU-DMPA-[Fe(DOPA $)_{3}$ ] is supposed to show complete stress relaxation. However, surprisingly this behavior does not appear (Figure 3a). We believe that there must be a synergistic effect between network reshuffling and formation of catechol$\mathrm{Fe}^{3+}$ crosslinks in this case. Iron ions have been known to be highly mobile throughout the material in cooperation with the network rearrangement [10]. When the specimen is stretched, the transverse contraction forces reduction of intermolecular distance. Accordingly, quite a few transient crosslinks are easily established among neighbor macromolecules and iron ions. Because (i) formation of additional crosslinks is faster than relaxation movement of the molecular chains and (ii) the higher crosslinking density corresponds to higher strength of the material (Figure 5), the measured stress increases with time after the initial drop due to conformation change (Figure 3a). With respect to dry HBPU-DMPA-[Fe(DOPA $\left.)_{3}\right]$ and the control HBPU-DMPA-DOPA, no dynamic bonds are available so that their stress relaxation behaviors resemble those of irreversibly bonded polymers. The stress
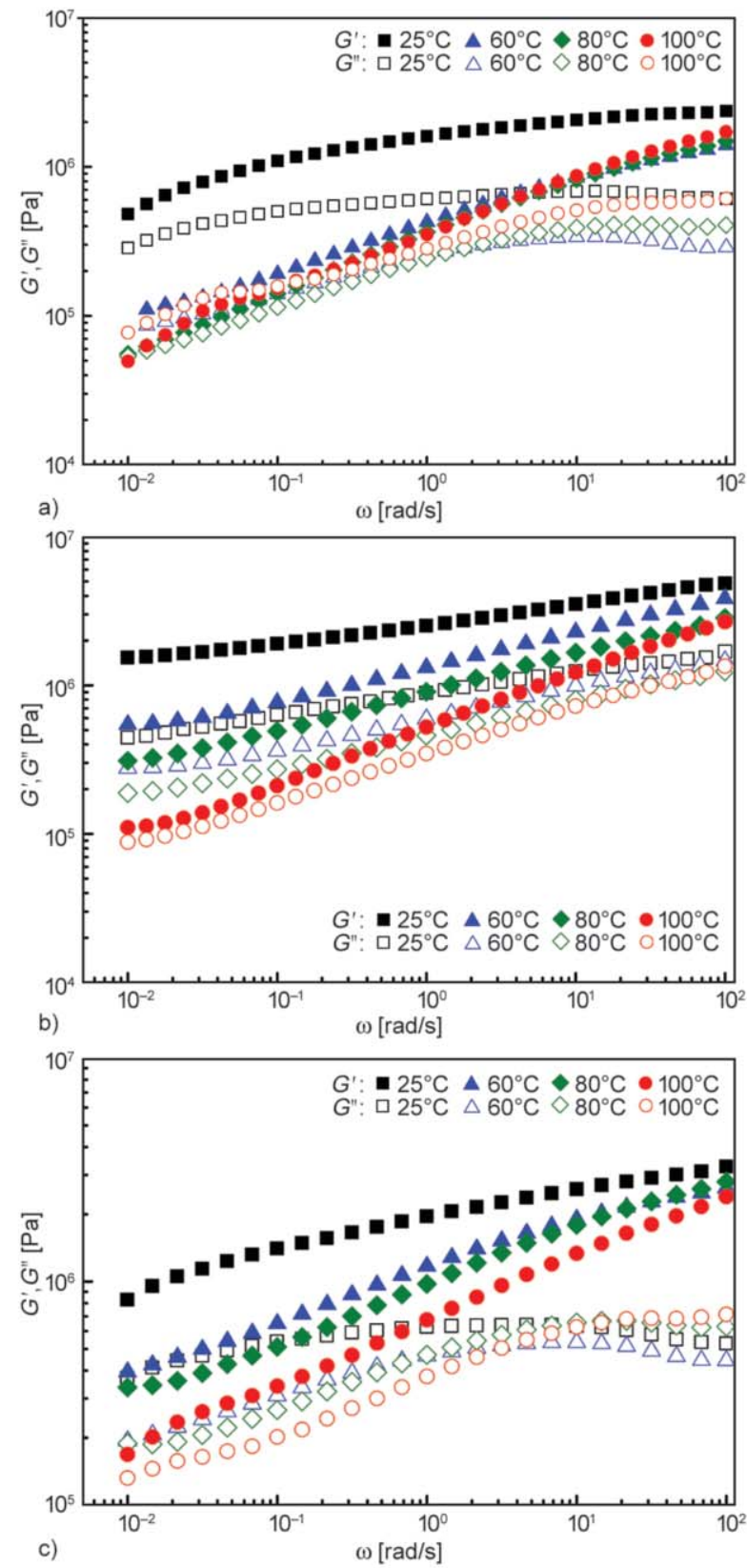

Figure 4. Storage shear modulus, $G^{\prime}$, and loss shear modulus, $G^{\prime \prime}$, as a functions of oscillatory frequency, $\omega$, for (a) HBPU-DMPA-[Fe(DOPA $\left.)_{3}\right]$ saturated by water $(\mathrm{pH}=9)$, (b) HBPU-DMPA-[Fe(DOPA $\left.)_{3}\right]$ that was firstly saturated by water $(\mathrm{pH}=9)$ and then dried, and (c) HBPU-DMPA-DOPA saturated by water $(\mathrm{pH}=9)$ 


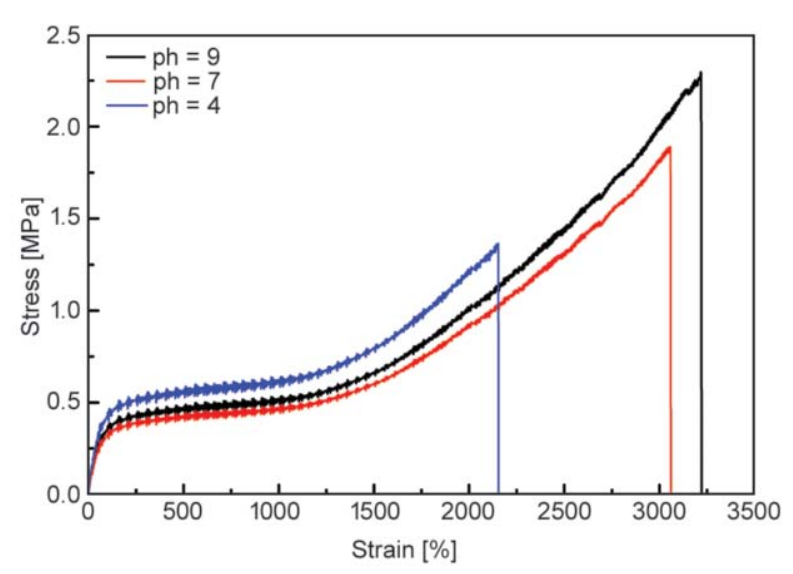

Figure 5. Tensile stress-strain curves of HBPU-DMPA$\left[\mathrm{Fe}(\mathrm{DOPA})_{3}\right]$ measured in water at $25^{\circ} \mathrm{C}$

rapidly declines to a stable value and then no longer changes with time (Figures $3 b$ and $3 c$ ).

To verify the analysis, a group of model experiments are designed as follows. Firstly, HBPU-DMPA$\left[\mathrm{Fe}(\mathrm{DOPA})_{3}\right]$ was compressed in a mold to simulate the Poisson's contraction induced during tensile stress relaxation test (refer to the Experimental part for more details). Next, Raman spectra of the compressed specimen were collected as a function of compression time (equivalent to stress relaxation time to some degree). As the band at $500 \sim 650 \mathrm{~cm}^{-1}$ originates from chelation of $\mathrm{Fe}^{3+}$ with oxygen atoms of catechol [15], the peak area is correlated with coordination number of catechol- $\mathrm{Fe}^{3+}$ bonds. Although the compressed specimens had to be taken out of the mold for the spectroscopic measurement, the plastic deformation produced by compression cannot be fully recovered, so that the obtained structural information still roughly describes the characteristics of the crosslinkages as expected.

It is seen from Figure 6 that the area of the Raman peak at $500 \sim 650 \mathrm{~cm}^{-1}$ of water saturated HBPUDMPA- $\left[\mathrm{Fe}(\mathrm{DOPA})_{3}\right]$ increases with a rise in compression time in the case of $\mathrm{pH}=7$ and 9. Moreover, the increment for $\mathrm{pH}=9$ is greater than that for $\mathrm{pH}=7$. Clearly, amount of the newly formed catechol- $-\mathrm{Fe}^{3+}$ crosslinkage increases with time at a constant $\mathrm{pH}$ value of 7 or 9 , and also increases with $\mathrm{pH}$ at a constant time. When $\mathrm{pH}=4$, the Raman peak area keeps unchanged within the time rage of interests, meaning the quantity of catechol- $\mathrm{Fe}^{3+}$ crosslinkage remains the same. The situation apparently coincides with the dry HBPU-DMPA-[Fe(DOPA) $)_{3}$ (Figure 6).
By comparing Figure 3 with Figure 6, it is reasonable to conclude that the Poisson's contraction created in the course of tensile stress relaxation test promotes formation of catechol- $\mathrm{Fe}^{3+}$ crosslinkage in water saturated HBPU-DMPA-[Fe(DOPA $\left.)_{3}\right]$. This is true especially for $\mathrm{pH}=9$. Considering the fact that the tris-coordinate catechol- $\mathrm{Fe}^{3+}$ crosslinks generate larger confinement than bis- and mono-catechol- $\mathrm{Fe}^{3+}$ coordination established at $\mathrm{pH}=7$ and 4 , the polymer exhibits more obvious self-strengthening effect with a rise in time under the circumstances. ${ }^{57} \mathrm{Fe}$ Mössbauer measurements of model complex of $\mathrm{Fe}[\mathrm{DOPA}]_{3}$ indicate that in the case of $\mathrm{pH}=9$, both tris- and bis-coordination bonds appear in anhydrous sample, while tris-coordination bonds predominate in water saturated version due to the dynamic coordination-dissociation of DOPA-iron interaction [10]. Compared to small molecular $\mathrm{Fe}[\mathrm{DOPA}]_{3}$, however, migration of iron ions in HBPU-DMPA-[Fe(DOPA) $)_{3}$ ] has to be hindered by the surrounding macromolecules even when water is present. Consequently, some iron ions can only establish bis-coordination with catechol for $\mathrm{pH}=9$ due to the kinetic factor. The coexistence of tris- and bis-catechol- $\mathrm{Fe}^{3+}$ crosslinks $[11,16]$ makes it possible for the polymer HBPUDMPA- $\left[\mathrm{Fe}(\mathrm{DOPA})_{3}\right]$ to increase its crosslinking density by converting bis-coordination into tris-coordination. So long as the intermolecular space is reduced, probability of collision of iron ions with catechol in the polymer is raised and more tris-coordination would be obtained (Figure 7).

For $\mathrm{pH}=7$, the crosslinks of HBPU-DMPA$\left[\mathrm{Fe}(\mathrm{DOPA})_{3}\right]$ is dominated by bis-catechol- $\mathrm{Fe}^{3+}$ co-

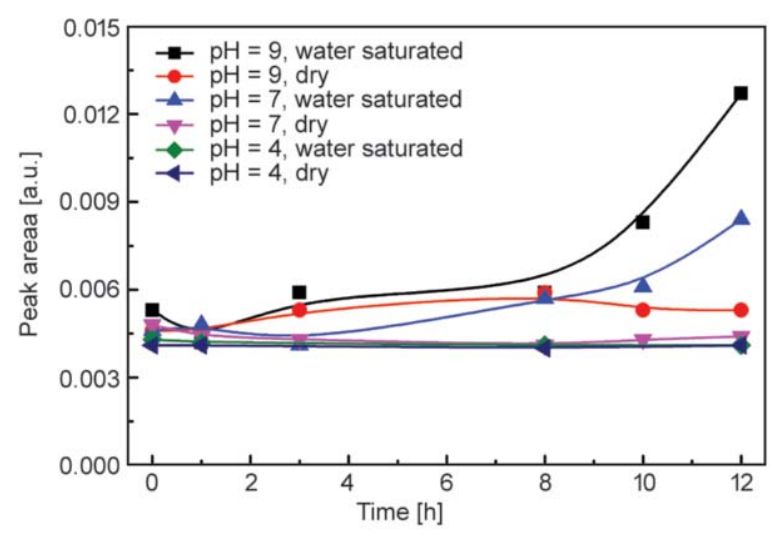

Figure 6. Area of the Raman peak at $500 \sim 650 \mathrm{~cm}^{-1}$ of HBPUDMPA-[Fe(DOPA $\left.)_{3}\right]$ versus compression time 


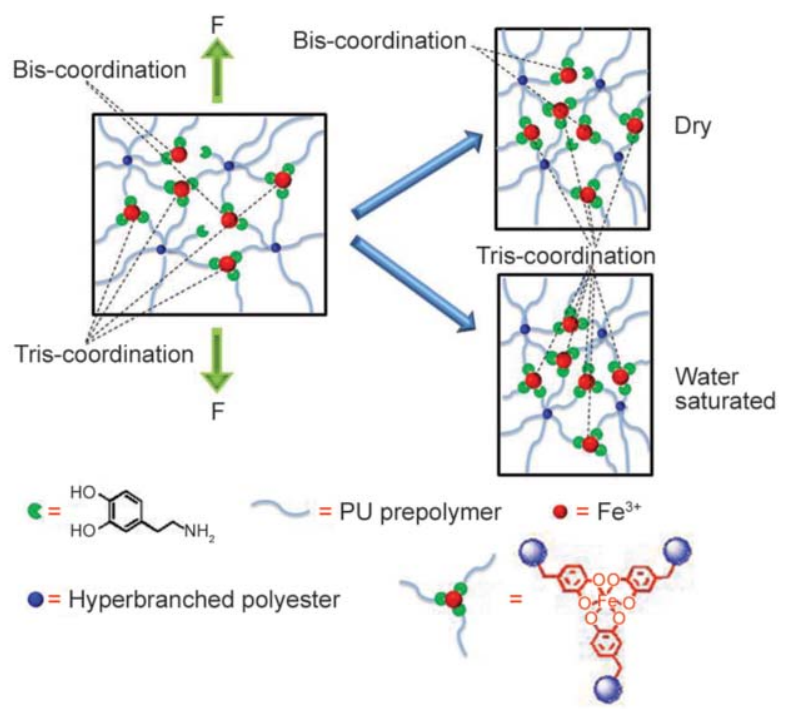

Figure 7. Change in coordination number of catechol- $\mathrm{Fe}^{3+}$ bonds of HBPU-DMPA-[Fe(DOPA $\left.)_{3}\right]$ in response to lateral contraction during tension $(\mathrm{pH}=9)$

ordination. Although amount of the crosslinks also increases, the improvement of material strength is less significant. Meantime, the compacted macromolecular chains do not contribute to formation of mono-catechol- $\mathrm{Fe}^{3+}$ coordination at $\mathrm{pH}=4$, as monocoordination does not necessarily involve aggregated molecules. Therefore, there is no detectable change in the corresponding curve of Figure 6. Since the intermolecular interaction is not enhanced, the specimen of water saturated HBPU-DMPA-[Fe(DOPA $\left.)_{3}\right]$ $(\mathrm{pH}=4)$ does not show stress intensification during stress relaxation test (Figure $3 \mathrm{a}$ ).

On the other hand, Figure 6 demonstrates that the catechol-iron interaction nearly does not vary in the dry HBPU-DMPA-[Fe(DOPA) 3 ]. Because the dynamic bonds are immobilized in the material, coordination number of catechol- $\mathrm{Fe}^{3+}$ bonds has to be unchanged regardless of mobility of iron ions (Figure 7). It agrees with the results of stress relaxation that the specimens behave like conventional polymers (Figure 3b).

In fact, stress intensification is not limited to HBPUDMPA-[Fe(DOPA $\left.)_{3}\right]$. When the iron is replaced by boron, the water saturated HBPU-DMPA-[B(DOPA $\left.)_{2}\right]$ $(\mathrm{pH}=9)$ also shows the same effect (Figure 8). There is no technique available for determining coordination status of boron in bulk material for the moment. Detailed investigation of the underlying mechanism has to be made in the future, but the result provides a useful reference for designing materials with similar property.

Nevertheless, it is worth noting that dynamically bonded polymers do not necessarily exhibit similar stress intensification manner. As mentioned in the Introduction, the stress applied to the polymers crosslinked by dynamic reversible bonds (like disulfide, $\mathrm{C}-\mathrm{ON}$ and aromatic Schiff base bonds) used to relax to zero [3-9]. It might be attributed to the fact that these polymers lack the mechanism of increasing crosslinks during stress relaxation.

\section{Conclusions}

The stress intensification observed during tensile stress relaxation test of HBPU-DMPA-[Fe(DOPA $\left.)_{3}\right]$ proves to result from increase of catechol-iron crosslinkages. Formation of the additional crosslinkages is so fast that relaxation of the chain segments are not ready to proceed. High $\mathrm{pH}$ (e.g. $\mathrm{pH}=9$ ) of the water used for saturating the polymer favors creation of more tris-coordination and hence more obvious the self-strengthening effect. The habit might find application in offshore engineering.

In a broad sense, the stress intensification is useful for improving durability of polymeric products for long-term usage as they would no longer flow under a fixed load like conventional versions but become stronger. Further efforts are needed to know more about the working principle, to enhance the effect, and to reproduce the property across different materials.

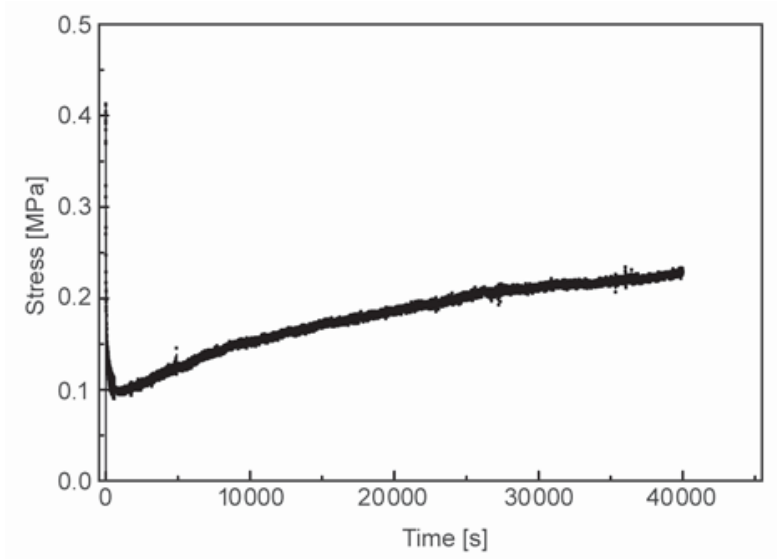

Figure 8. Tensile stress relaxation behavior of water saturated HBPU-DMPA-[B(DOPA $\left.)_{2}\right](\mathrm{pH}=9)$. Temperature: $25^{\circ} \mathrm{C}$. 


\section{Acknowledgements}

The authors thank the support of the Natural Science Foundation of China (Grants: 51273214 and 51333008), the Natural Science Foundation of Guangdong (Grant: S2013020013029), the Science and Technology Program of Guangzhou (Grant: 2014J4100121), and the Basic Scientific Research Foundation in Colleges and Universities of Ministry of Education of China (Grants: 15lgzs103 and 14lgqt01).

\section{References}

[1] Manson J. A., Sperling L. H.: Polymer blends and coposites. Springer, New York (1976).

[2] Sperling L. H.: Introduction to physical polymer science. Wiley, Hoboken (2006).

[3] Pepels M., Filot I., Klumperman B., Goossens H.: Selfhealing systems based on disulfide-thiol exchange reactions. Polymer Chemistry, 4, 4955-4965 (2013). DOI: $10.1039 / \mathrm{C} 3 \mathrm{PY} 00087 \mathrm{G}$

[4] Lei Z. Q., Xie P., Rong M. Z., Zhang M. Q.: Catalystfree dynamic exchange of aromatic Schiff base bonds and its application to self-healing and remolding of crosslinked polymers. Journal of Materials Chemistry A, 3, 19662-19668 (2015). DOI: $10.1039 / C 5 T A 05788 \mathrm{D}$

[5] Lei Z. Q., Xiang H. P., Yuan Y. J., Rong M. Z., Zhang M. Q.: Room-temperature self-healable and remoldable cross-linked polymer based on the dynamic exchange of disulfide bonds. Chemistry of Materials, 26, 20382046 (2014). DOI: $10.1021 / \mathrm{cm} 4040616$

[6] Xiang H. P., Qian H. J., Lu Z. Y., Rong M. Z., Zhang M. Q.: Crack healing and reclaiming of vulcanized rubber by triggering the rearrangement of inherent sulfur crosslinked networks. Green Chemistry, 17, 4315-4325 (2015). DOI: 10.1039/C5GC00754B

[7] Zhang Z. P., Rong M. Z., Zhang M. Q.: Room temperature self-healable epoxy elastomer with reversible alkoxyamines as crosslinkages. Polymer, 55, 3936-3943 (2014).

DOI: $10.1016 /$ j.polymer.2014.06.064

[8] Yuan C. E., Rong M. Z., Zhang M. Q.: Self-healing polyurethane elastomer with thermally reversible alkoxyamines as crosslinkages. Polymer, 55, 1782-1791 (2014)

DOI: $10.1016 /$ j.polymer.2014.02.033
[9] Zhang Z. P., Lu Y., Rong M. Z., Zhang M. Q.: A thermally remendable and reprocessable crosslinked methyl methacrylate polymer based on oxygen insensitive dynamic reversible $\mathrm{C}-\mathrm{ON}$ bonds. RSC Advances, 6, 63506357 (2016).

DOI: $10.1039 / C 5 R A 22275 C$

[10] Xia N. N., Xiong X. M., Wang J., Rong M. Z., Zhang M. Q.: A seawater triggered dynamic coordinate bond and its application for underwater self-healing and reclaiming of lipophilic polymer. Chemical Science, 7, 2736-2742 (2016).

DOI: $10.1039 / C 5 S C 03483 C$

[11] Holten-Andersen N., Harrington M. J., Birkedal H., Lee B. P., Messersmith P. B., Lee K. Y. C., Waite J. H.: pHinduced metal-ligand cross-links inspired by mussel yield self-healing polymer networks with near-covalent elastic moduli. Proceedings of the National Academy of Sciences of the United States of America, 108, 26512655 (2011). DOI: $10.1073 /$ pnas. 1015862108

[12] Roberts M. C., Hanson M. C., Massey A. P., Karren E. A., Kiser P. F.: Dynamically restructuring hydrogel networks formed with reversible covalent crosslinks. Advanced Materials, 19, 2503-2507 (2007).

DOI: 10.1002/adma.200602649

[13] Bose R. K., Hohlbein N., Garcia S. J., Schmidt A. M., van der Zwaag S.: Connecting supramolecular bond lifetime and network mobility for scratch healing in poly(butyl acrylate) ionomers containing sodium, zinc and cobalt. Physical Chemistry Chemical Physics, 17, 1697-1704 (2015).

DOI: $10.1039 / \mathrm{C} 4 \mathrm{CP} 04015 \mathrm{E}$

[14] Bode S., Enke M., Bose R. K., Schacher F. H., Garcia S. J., van der Zwaag S., Hager M. D., Schubert U. S.: Correlation between scratch healing and rheological behavior for terpyridine complex based metallopolymers. Journal of Materials Chemistry A, 3, 22145-22153 (2015). DOI: $10.1039 / \mathrm{C} 5 \mathrm{TA} 05545 \mathrm{H}$

[15] Michaud-Soret I., Andersson K. K., Que Jr. L., Haavik J.: Resonance Raman studies of catecholate and phenolate complexes of recombinant human tyrosine hydroxylase. Biochemistry, 34, 5504-5510 (1995). DOI: $10.1021 / \mathrm{bi00016a022}$

[16] Sever M. J., Wilker J. J.: Absorption spectroscopy and binding constants for first-row transition metal complexes of a DOPA-containing peptide. Dalton Transactions, 2006, 813-822 (2006). DOI: $10.1039 / \mathrm{B} 509586 \mathrm{G}$ 